\title{
A circularly polarized harmonic-rejecting antenna for wireless power transfer applications
}

\author{
Omar A. Barrera, Dong Hyun Lee, Nguyen Manh Quyet, \\ Viet Hoang-The, and Hyun Chang Park ${ }^{\mathrm{a})}$ \\ Division of Electronics and Electrical Engineering, Dongguk University-Seoul, \\ Seoul, 100-715, Korea \\ a)hcpark@dongguk.edu
}

\begin{abstract}
A dual-feed circularly polarized antenna with built-in harmonic-rejection capabilities is presented for the first time. The square patch antenna has been designed at $5.8 \mathrm{GHz}$ and incorporates both circular polarization and harmonic-rejection in a single design, thus eliminating the need of a low-pass filter. A comparison among the proposed antenna, a simple square patch antenna, and a truncated square patch antenna is performed to evaluate the capabilities of the proposed design. The realized antenna exhibits a reflection coefficient of $-1.92 \mathrm{~dB}$ at the second harmonic frequency of $11.6 \mathrm{GHz}$, and circular polarization for elevation angles in the range of $-60^{0}$ to $60^{\circ}$, well suited for a rectenna in wireless power transfer applications.
\end{abstract}

Keywords: antenna, rectenna, circular polarization, harmonic rejection, wireless power transfer, dual feed

Classification: Microwave and millimeter wave devices, circuits, and systems

\section{References}

[1] J. A. Hagerty, F. B. Helmrecht, W. H. McCalpin, R. Zane and Z. B. Popovic: IEEE Trans. Microw. Theory Tech. 52 (2004) 1014.

[2] Y. Suh, C. Wang and K. Chang: Electron. Lett. 36 (2000) 600.

[3] T. C. Yo, C. M. Lee, C. M. Hsu and C. H. Luo: IEEE Trans. Antennas Propag. 56 (2008) 882.

[4] Y. Park, S. Han and T. Itoh: IEEE Antennas Wireless Propag. Lett. 3 (2004) 52.

[5] R. Dehbashi, Z. Atlasbaf and K. Forooraghi: IEEE Antennas Wireless Propag. Lett. 5 (2006) 395.

[6] G. Kumar and K. P. Ray: Broadband Microstrip Antenna (Artech House Inc., London, 2003) 123.

[7] T. D. Nguyen, D. H. Lee and H. C. Park: IEEE Antennas Wireless Propag. Lett. 10 (2011) 403.

[8] K. C. Gupta, R. Garg, I. Bahl and P. Bhartia: Microstrip Lines and Slotlines (Artech House, Boston, 1996) 2nd ed. 135. 
[9] H. Takhedmit, L. Cirio, B. Merabet, B. Allard, F. Costa, C. Vollaire and O. Picon: Electron. Lett. 46 (2010) 811.

[10] M. S. Toubet, A. E. Ahmad, R. Chantalat, M. Hajj, E. Arnaud, B. Jecko, T. Monediere, C. Boustie and B. Palacin: Int. J. Antennas Propag. 2012 (2012) 8.

[11] B. Strassner and K. Chang: IEEE Trans. Microw. Theory Tech. 51 (2003) 1548.

\section{Introduction}

A rectifying antenna (or rectenna) plays an important role in wireless power transfer technologies [1]. A rectenna typically consists of five components, a receiving antenna, a low-pass filer, a rectifier circuit, a dc-pass filter, and a load.

The receiving antenna is one of the main consideration blocks in the rectenna design. Several features to improve the overall performance of a rectenna have been applied to the antenna, resulting in circularly polarized (CP) antennas and harmonic-rejecting antennas. When it comes to CP antennas, truncated square patch antennas [2] have become very popular due to the easy design and fabrication. Circular patches with unbalanced slots for circular polarization have also been reported [3]. These CP antennas are typically followed by a low-pass filter to stop diode-generated harmonics from being re-radiated by the antenna. On the other hand, harmonic-rejecting antennas usually result from modifications within the radiator to increase the reflection at the harmonic frequencies, thus eliminating the need of a separate low-pass filter $[4,5]$. Such modifications to the radiator, however, make it very difficult for the antenna to radiate circularly polarized waves.

In order to address these challenges, a circularly polarized antenna with built-in harmonic-rejection capabilities is presented. A dual-feed square patch antenna was first designed for circular polarization. A quarterwavelength stub was then attached to one of the feed lines outside the radiator patch to suppress re-radiation of the second harmonic. The proposed configuration eliminates the need of a low-pass filter while maintaining $\mathrm{CP}$ capabilities in a single antenna element.

\section{Design and analysis}

The $5.8 \mathrm{GHz}$ square patch antenna was designed using Ansoft's High Frequency Structure Simulator (HFSS) for a Taconic substrate with a dielectric constant of 2.2. The final proposed design is shown in Fig. 1. Initially, two microstrip quarter-wavelength transformers with equal length of $10.2 \mathrm{~mm}$ were attached to two neighboring edges of the radiator and fed a $100 \Omega$ microstrip line. The guided wavelength is $\lambda_{g}=\frac{\lambda_{0}}{\sqrt{\varepsilon_{e f f}}}=40.8 \mathrm{~mm}$ at $5.8 \mathrm{GHz}$, where $\lambda_{0}$ is the free-space wavelength and the effective dielectric constant is $\varepsilon_{\text {eff }}=\frac{\varepsilon_{r}+1}{2}$. No phase difference exists at the points where the quarter-wavelength transformers join the $100 \Omega$ line. A probe feed was then placed on the $100 \Omega$ line 
in such a way that the length to one end of the $100 \Omega$ line is $\lambda_{g} / 4$ longer than the length to the other end, thereby providing the $90^{\circ}$ phase difference required to achieve circular polarization. This configuration also provides a $50 \Omega$ properly matched output since the two $100 \Omega$ lines are in parallel [6]].

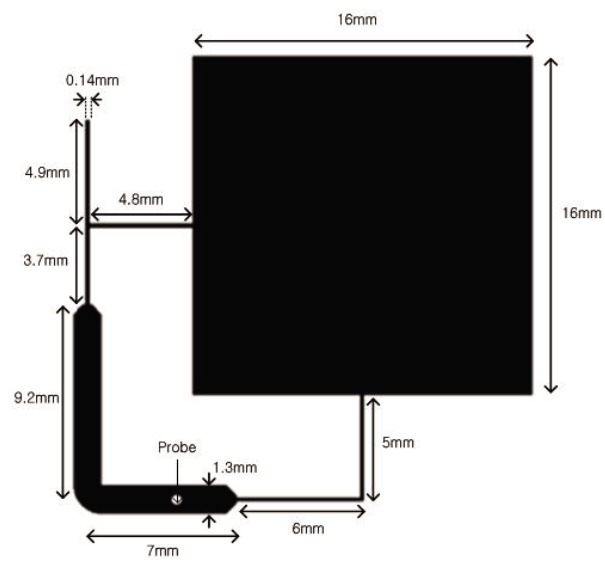

Fig. 1. Proposed Antenna.

Finally, a $5.1 \mathrm{~mm}$ stub (quarter-wavelength at $11.6 \mathrm{GHz}$ ) was attached in the middle of the impedance transformer on the longer path to the probe feed. Fig. 2 shows the surface current distribution of the antenna and the feed lines. At $11.6 \mathrm{GHz}$, surface current is concentrated at the stub while very low current is observed in the radiator, indicating efficient rejection of the second harmonic. This operation can be further explained using an opencircuit-terminated series stub in a transmission line model $[7,8]$ as shown in Fig. 3.
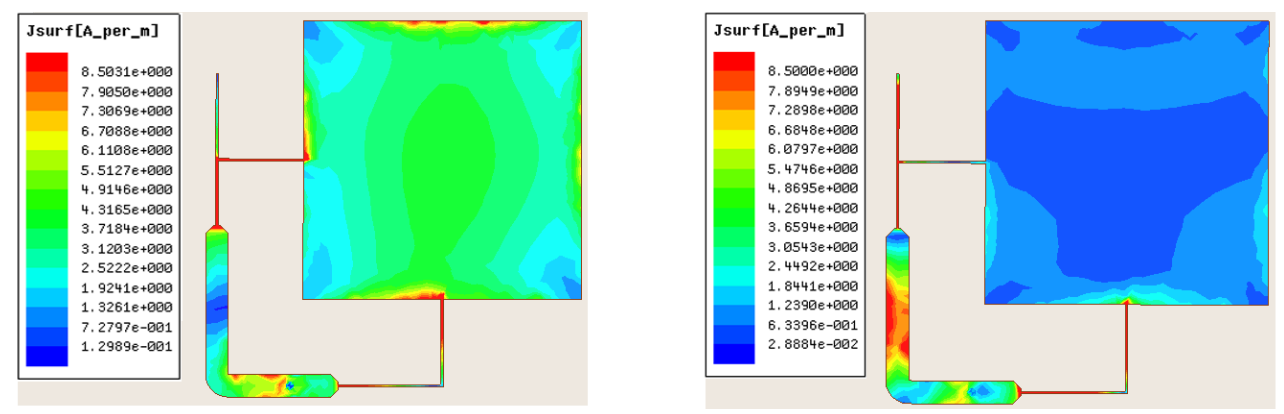

Fig. 2. The current distribution of antenna at (a) $5.8 \mathrm{GHz}$ and (b) $11.6 \mathrm{GHz}$.

Only at $11.6 \mathrm{GHz}$, the length of the stub $L_{s}$ equals $\lambda_{g} / 4$, and the stub works as a quarter-wavelength transmission line terminated in an open circuit. Therefore, the stub behaves as an short-circuited series stub, reflecting the unwanted $11.6 \mathrm{GHz}$ second harmonic generated by the rectifier circuit of the rectenna [9].

Using another stub attached to the other impedance transformer on the shorter path would lead to a slightly better second harmonic rejection. In 


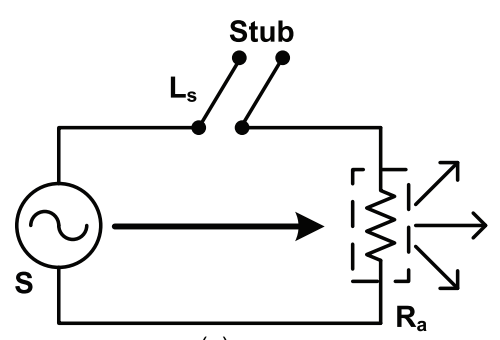

(a)

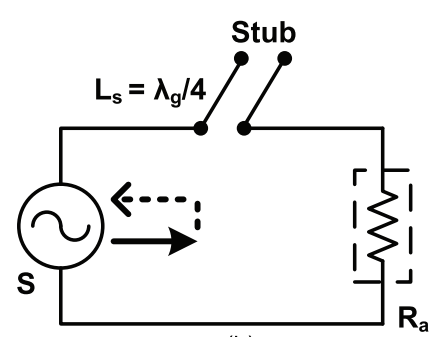

(b)

Fig. 3. Transmission line model for the antenna at (a) fundamental frequency of $5.8 \mathrm{GHz}$ and (b) second harmonic frequency of $11.6 \mathrm{GHz}$ (with source $\mathrm{S}$ and antenna resistance $R_{a}$ ).

this case, however, the impedance at both junctions between the quarterwavelength transformer and the $100 \Omega$ line becomes identical. As a result, the input impedance of each arm at the feed point becomes quite different, resulting in much degraded axial ratio as shown in Fig. 4.

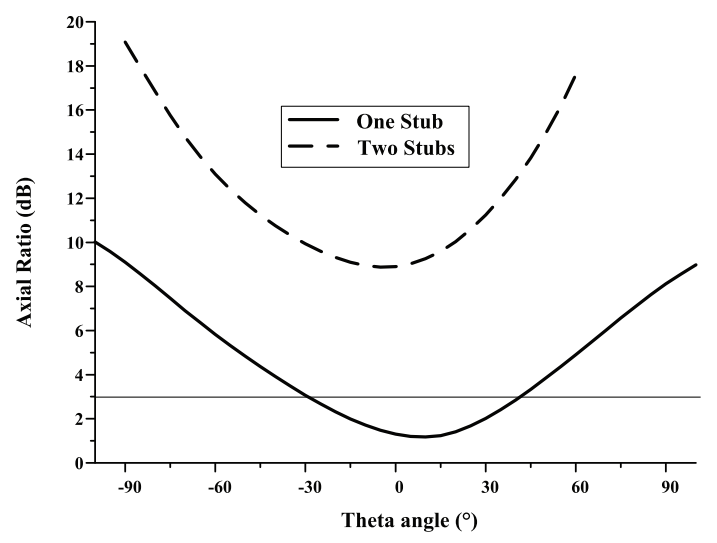

Fig. 4. Simulated axial ratio of the antenna with one or two stubs for second harmonic rejection.

The final lengths of the impedance transformers and the harmonicrejection stub were then optimized as shown in Fig. 1 to achieve maximum performance in terms of reflection coefficient and axial ratio.

Along with the proposed patch antenna, a simple square patch antenna and a truncated square patch antenna with the same dimensional parameters were compared. As shown in Fig. 5, reflection coefficients of the proposed antenna, truncated antenna and square antenna were $-3.8 \mathrm{~dB},-17.3 \mathrm{~dB}$ and $-17.5 \mathrm{~dB}$ respectively, showing clear rejection of the second harmonic at 11. $6 \mathrm{GHz}$ by the proposed antenna. The axial ratio characteristics of the proposed antenna indicates circular polarization in a symmetric fashion for a much wider angular aperture of $120^{\circ}$ over those of previous reports [10, 11], generating circular polarization not only in the broadside direction, but also over a broad angular range of beam as shown in Fig. 6 . 


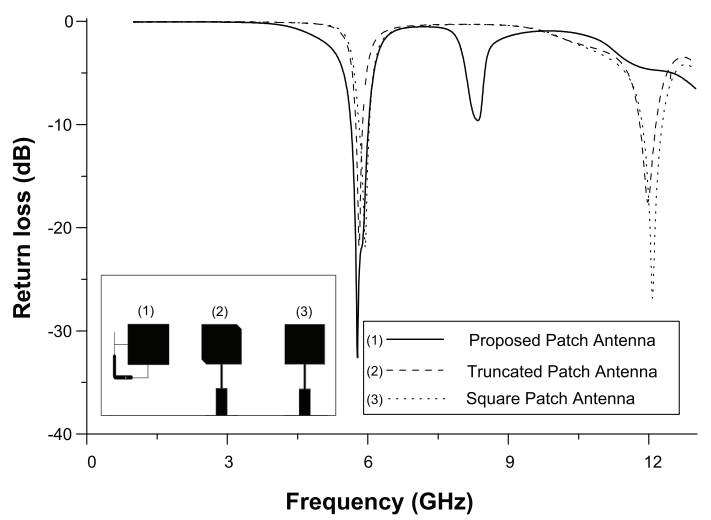

Fig. 5. Simulated reflection coefficient of the patch antennas.

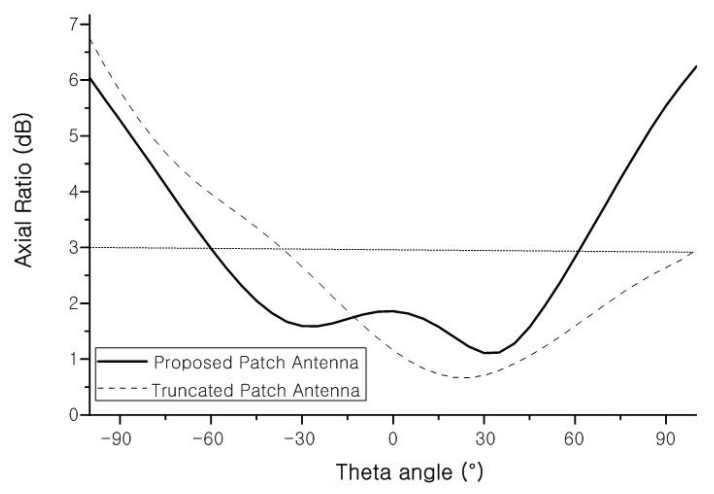

Fig. 6. Simulated axial ratio.

\section{Result and discussions}

Fig. 7 (a) shows the photograph of the proposed antenna after fabrication. Reflection coefficients of the three patch antennas were measured using an HP 8719A network analyzer. As shown in Fig. 7 (b), all the antennas show good reflection coefficient at the fundamental frequency of $5.8 \mathrm{GHz}$. However, reflection coefficients of the square patch antenna and the truncated square patch antenna are very low at the second harmonic, $-6.52 \mathrm{~dB}$ and $-10.1 \mathrm{~dB}$ respectively. Therefore, a low-pass filter would be required for a rectenna design using these antennas. On the other hand, the proposed antenna substantially increases the reflection coefficient of the second harmonic to $-1.92 \mathrm{~dB}$, relieving the necessity of a low-pass filter.

The Bandwidth (BW) of the proposed patch antenna at the fundamental frequency is also twice as big as that of the simple patch antenna and considerably larger than that of the truncated patch antenna, with BW of $400 \mathrm{MHz}, 200 \mathrm{MHz}, 270 \mathrm{MHz}$, respectively.

Fig. 8 shows the simulated radiation pattern of the proposed antenna at 5.8 GHz. Both the E-plane and H-plane patterns are stable and symmetrical. Moreover, the axial ratio is kept below $2 \mathrm{~dB}$ within the half-power beamwidth of $80^{\circ}$. These radiation characteristics are desired for wireless power transfer applications [11]. 


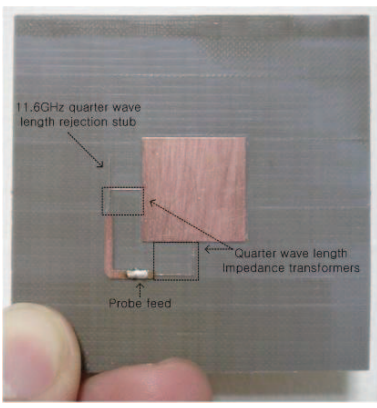

(a)

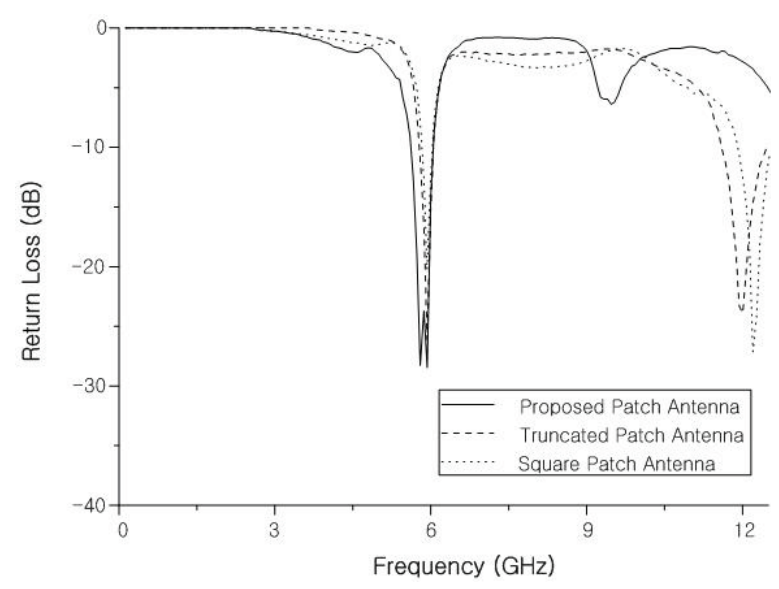

(b)
Fig. 7. (a) Fabricated antenna of the proposed design and (b) measured reflection coefficients of the patch antennas.

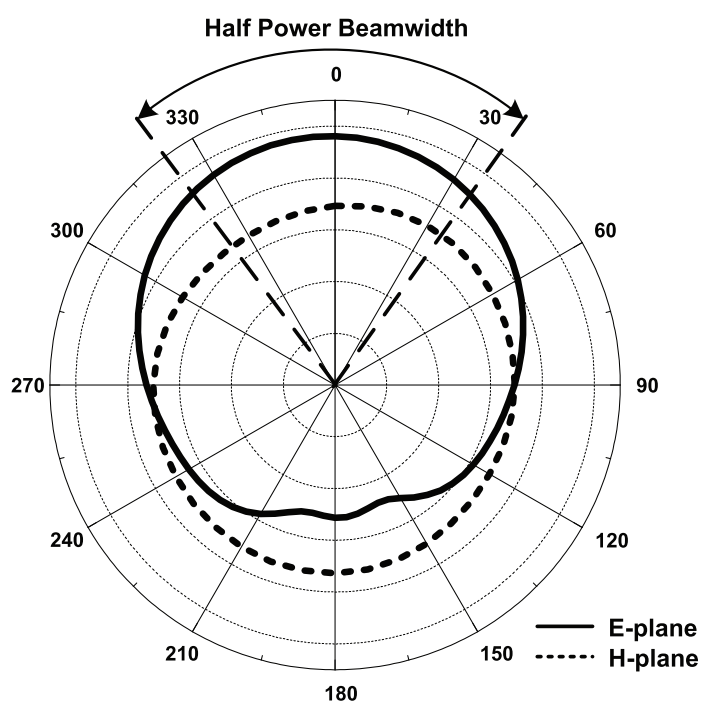

Fig. 8. Radiation patterns in E- and H-plane at $5.8 \mathrm{GHz}$.

\section{Conclusion}

A dual-feed circularly polarized antenna with built-in harmonic-rejection capabilities was demonstrated for the first time. The antenna achieved reflection coefficient of $-1.92 \mathrm{~dB}$ at the second harmonic frequency of $11.6 \mathrm{GHz}$, and circular polarization in the wide range of elevation angles from $-60^{0}$ to $60^{0}$ in a single, compact configuration. This antenna is well suited for a rectenna in wireless power transfer applications.

\section{Acknowledgments}

This work was supported by the National Research Foundation of Korea (NRF) under the Basic Science Research Program founded by the Ministry of Education, Science and Technology (2010-0023571). 